\title{
Experiencing the Elements - User Study with Natural Material Probes
}

\author{
Jonna Häkkila ${ }^{1}$, Yun $\mathrm{He}^{2}$, and Ashley Colley ${ }^{2(\bowtie)}$ \\ ${ }^{1}$ Faculty of Art and Design, University of Lapland, Rovaniemi, Finland \\ jonna.hakkila@ulapland.fi \\ ${ }^{2}$ CIE, University of Oulu, Oulu, Finland \\ \{yun. he, ashley.colley\}@cie.fi
}

\begin{abstract}
In this paper, we present the first systematic user study exploring the user experience and perceptions towards different natural materials - water, ice, stone, sand, fire, wind and soup bubbles. By trying out different materials, participants $(n=16)$ expressed their associations and perceptions, rated different qualities of the materials, and described their impressions through product reaction cards. Our findings reveal for example that light weight and ease of movement are perceived as central qualities when inspiring and fun elements are sought for. This exploratory study shines light on user experiences with natural elements, and provides an experimental grounding for naturalistic tangible user interface design. Material qualities in tangible user interface design create a subtle, but critical part of the user experience.
\end{abstract}

Keywords: Material qualities - User experience - Tangible user interfaces • Design · User studies

\section{Introduction}

In [1], Hassenzahl defines user experience (UX) as "a momentary, primarily evaluative feeling (good-bad) while interacting with a product or service”. User experience consists of both utilitarian and a hedonic aspect [2]. Whereas the utilitarian side typically dominates in the overall motivation for application design, in constructing the user interface, hedonic aspects are important to consider in order to create pleasurable and engaging user experiences. When designing tangible user interfaces, material qualities are an integral part of the holistic experience.

Material qualities of physical objects have been thoroughly considered in areas such as art, industrial design and mechanics, but research on the material's role in interactive systems has been quite sporadic, leaving much to explore. Especially, the use of natural materials has so far been little researched. As tangible user interfaces (TUIs) can make use of the human senses in a richer and more multidimensional way than conventional digital user interfaces (UIs) [3], it is of interest to explore the different qualities that are perceived and associated with different physical materials.

In this paper, we take steps towards systematic exploration of natural materials. We are especially interested of these because of the new possibilities they provide for the design for tangible user interfaces, aesthetics and rich user experiences, and offer 
potential for user interfaces and application linked with, e.g., sustainable values or interactive environmental installations.

\section{Prior Art and Positioning of Our Work}

Prior art has demonstrated the use of different natural materials in different types of interactive systems, often designed for playful purposes or as part of a larger, monumental installation. Döring et al. have demonstrated interaction with soap bubbles [4], Virolainen et al. have shown touch screen interaction with an ice wall [5] whilst water based input and output has been used e.g. in [6, 7]. Heavy stone based input [8], and air based haptic feedback [9] have also been demonstrated. Rydarowski et al. [10] present an installation, where individually controlled CPU fans are used to move paper clips with air pressure.

The user experience findings reflect the curiosity [7], aesthetics [5], and playful nature of the interaction $[5,8]$. With water related interactive systems, the pleasant sensation the cool water creates against the skin has been highlighted by users [7,8], and spilling water has been reported to offer a more powerful UI feedback than a virtual one [6].

Whereas examples of the use of natural materials in TUIs exist, differing from us, the earlier research has reported the user perceptions focusing each time on a single system without a systematic comparison between different tangible elements. Moreover, the focus of the prior art has been more on the proof-of-concept level installations rather than on explicit investigation of the user experience aspects. A few explorations of different material qualities exist, as in [11] in the context of fashion, fabric and shape-change, but to the best of our knowledge, not in the domain of natural materials.

\section{Study Methodology}

\subsection{Material Probes}

In our user study, we applied the material probes method introduced in [12]. Material probes for seven (7) natural materials were created. The materials were selected to have as wide a range of physical properties as possible, in order to elicit a full range of perceptions from our test participants when interacting with the probes. The material probes are shown in Fig. 1 and consisted of:

- Water, contained in a small bowl.

- Stone in the form of 3 smooth rocks.

- Soap bubbles, created as required from a soap solution container and wand.

- Ice, in the form of a tennis ball sized block.

- Sand, as a small pile on a plate.

- Wind, created as needed from a small desk fan.

- Fire, in the form of a candle flame. 


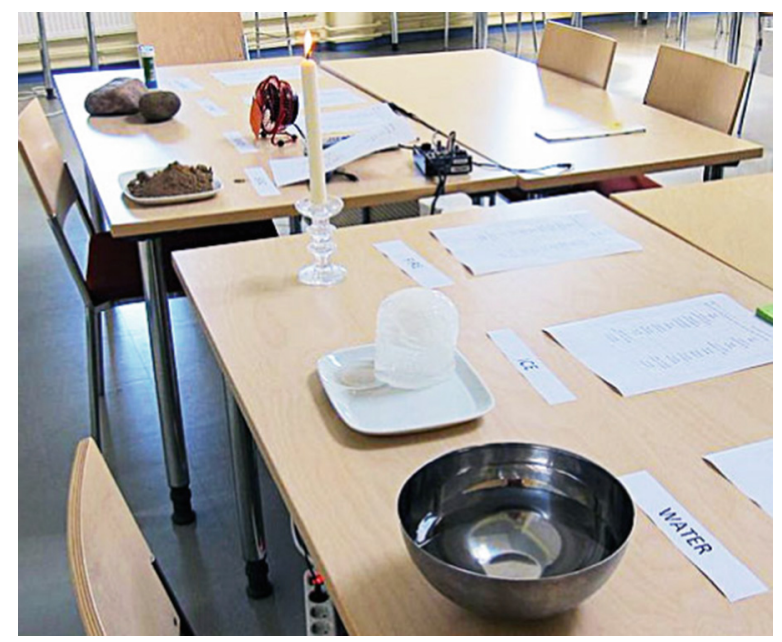

Fig. 1. User study set-up.

\subsection{User Study Procedure}

Our user study included an open think-aloud method, subjective rating of qualities and two tasks that required users to associate the materials with objects or activities. Additionally we utilized the product reaction cards method adapted from [13]. Each study session lasted for approximately $60 \mathrm{~min}$ and the study procedure was as follows:

- Completing a background questionnaire.

- Interacting with each material probe in turn and giving a free form description of ideas, thoughts and associations the material created.

- Selection of a favorite material (see Fig. 2)

- Rating the materials on a 7-point Likert scale against the criteria: Controllable, Calm, Inspiring and Overall preference.

- Connecting each material with digital artifacts, e.g. such as an alarm clock. Here, multiple artifacts could be connected with each material, and participants could leave some material unused (see Fig. 5).

- Connecting each material with activities and thoughts, e.g. such as organizing files. Again, multiple activities could be connected with each material, and participants could leave some material unused (see Fig. 6).

- For each material, selecting 3 adjectives from a list of 20 that best describe the material. Here following a Product Reaction Cards (PRC) based methodology [13]. Table 1 contains the adjectives that were available.

The order in which participants interacted with the materials was counterbalanced to avoid any effects due to the presentation order.

Altogether 16 participants ( 5 female, 11 male) took part in the study. The age range of the participants was 18-24:13\%, 25-29:37 \%, 30-39:44\% and 40-49:6\%. The participants were recruited through advertising on university email lists, and through personal networks. Although participants predominantly represented university 
Table 1. Adjectives used in product reaction card method. Participants selected three (3) that best described each material.

\begin{tabular}{l|l|l|l}
\hline Fast & Slow & Inconsistent & Consistent \\
\hline Responsive & Rigid & Uncontrollable & Controllable \\
\hline Fun & Serious & Unpleasant & Pleasant \\
\hline Restful & Stressful & Boring & Exciting \\
\hline Approachable & Unapproachable & Frustrating & Inspiring \\
\hline
\end{tabular}

students, they came from various backgrounds with several different nationalities being represented. No UX professionals or UI designers were included.

\section{Results - Perceptions of Different Materials}

\subsection{Favourite Material}

The materials the participants selected as favorite are shown in Fig. 2. Water was clearly the favorite material with almost half of the participants (7/16) selecting it as their favorite. Here, many of the participants highlighted the relaxing nature of water, for example, "Water is comfortable and controllable to interact with. I can do it for a long time, just like swimming." (User \#3).

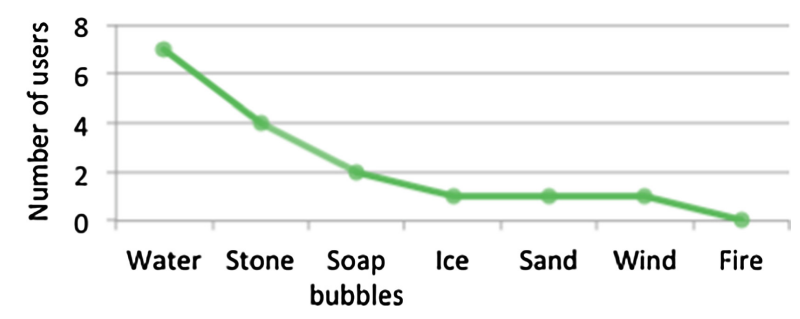

Fig. 2. Number of users preferring each material $(n=16)$

\subsection{Subjective Ratings and Product Reaction Cards}

When asked to subjectively rate their perceptions of the materials (Fig. 3), users considered fire to be the least controllable and least calm material. Several users commented on the danger of fire, "It is hot and untouchable. It hurts the hand. It is difficult to control and use." (User \#9). Water and Ice were perceived as two of the calmest and most inspiring elements. For both materials issues related to cleanliness and transparency were voiced e.g. "Transparent and clean" (User \#9, water), "Cold, beautiful and transparent” (User \#10, ice).

Examining the results from the Product Reaction Cards method (Fig. 4) provides congruent findings e.g. with fire and wind being considered uncontrollable by many 


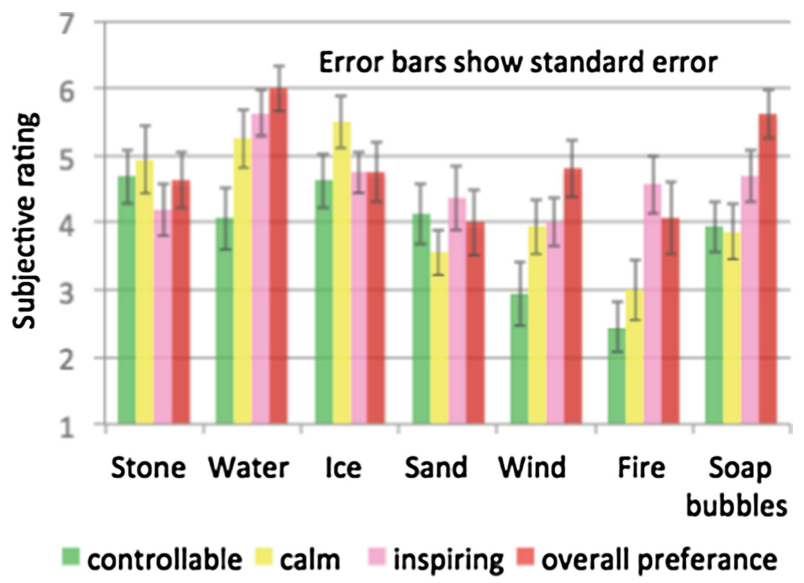

Fig. 3. Subjective ratings for each material

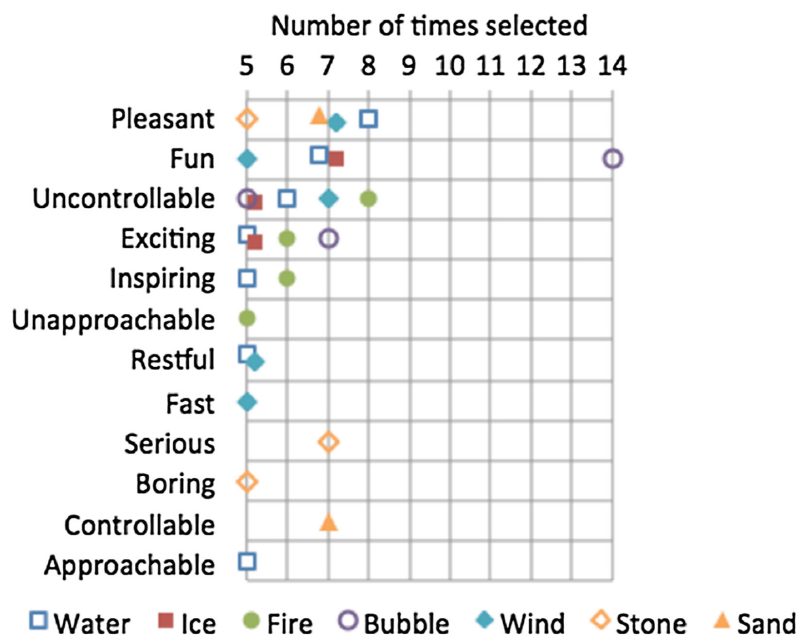

Fig. 4. Most frequently chosen adjectives for describing each material from Product Reaction Cards ( $N=16$ with 3 word selections per user).

users. Clearly soap bubbles are considered as fun, with all but 2 users (14/16) selecting this adjective.

\subsection{Material Associations}

The test participants associations between the materials and digital artifacts and activities are presented in Figs. 5 and 6 respectively. Whilst some of the strong associations are obvious, e.g. the association between the pairs ice - fridge and water - 


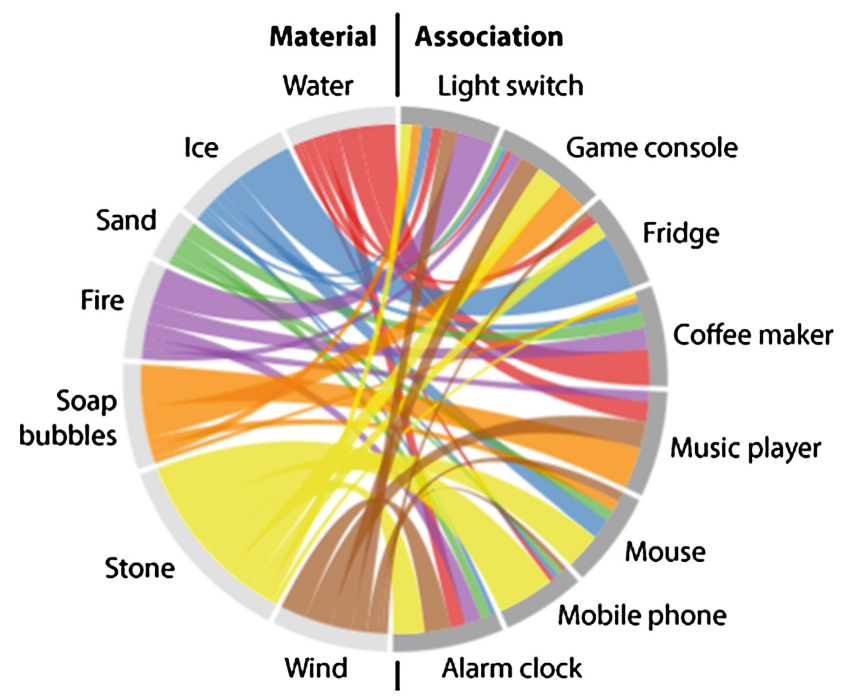

Fig. 5. Association between materials and digital artifacts. (The thickness of the base of each connection represents the number of users selecting that association).

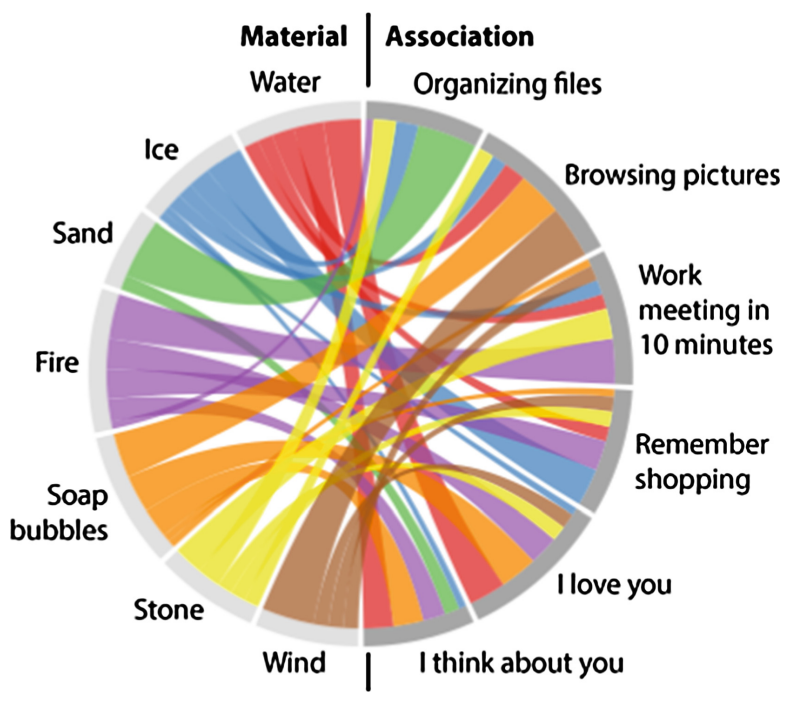

Fig. 6. Association between materials and activities/thoughts

coffee maker, other such as stone - mobile phone are less easily explained. Overall the high selection frequency of stone and low frequency of sand may be interesting to explore further. 
In general the association of the given activities with materials is rather even, with most activities being associated to some extent with all of the materials. One clear exception is that of sand, which was almost exclusively associated with organizing files.

\section{Discussion and Conclusions}

\subsection{Perceiving Materials}

In this paper, we have reported the first user study seeking to systematically explore the user experiences perceived with different natural materials, investigated by using material probes. Based on the PRC results, it seems that lucid and light materials water, wind and bubbles - are perceived as generally more playful, pleasant and fun. These materials also gained the highest ratings when the participants assessed in numerical scale how much they liked each material. Their light weight and ease of movement are perceived as central qualities when inspiring and fun elements are sought for. Although inspiring, fire was perceived uncontrollable and, based on the observations and comments, provoking reactions of respect and even fear.

Solid materials - stone, sand and ice - were perceived as more controllable and mostly pleasant. These were also the only materials that were associated with the task of 'organizing' (see Fig. 4). Whereas these associations are somewhat unsurprising, they confirm how materials that are quantifiable and behave less erratically are more easily associated with tasks that require control. This aligns with the findings reported by [8], where it is concluded that metaphors and interaction styles that match the physical characteristics of the interaction control should be utilized.

\subsection{Limitations of the Study and Methodological Observations}

In our study, we generally observed that people paid a lot of attention to each material many spent a long time interacting with the materials in the tasks, and sometimes also came back to them. The test sessions generally took more time than we had first expected.

We acknowledge that our study is limited by the small sample size of participants. However, we believe that our research is valuable in its attempt to conduct a comparable study on different user experience qualities of natural materials. The laboratory type context was disconnected from any specific domains or use cases, and hence we believe provided a good environment for obtaining baseline results.

\section{References}

1. Hassenzahl, M.: User experience (UX): towards an experiential perspective on product quality. In Proceedings of IMH 2008. ACM, pp. 11-15 (2008)

2. Hassenzahl, M., Tractinsky, N.: User experience - a research agenda. Behav. Inf. Technol. 25(2), 91-97 (2006) 
3. Ishii, H., Ullmer, B.: Tangible bits: towards seamless interfaces between people, bits and atoms. In: Proceedings of CHI 1997. ACM Press, pp. 234-241 (1997)

4. Döring, T., Sylvester, A., Schmidt, A.: Exploring material-centered design concepts for tangible interaction. Extended Abstracts CHI 2012. ACM Press, pp. 1523-1528 (2012)

5. Virolainen, A., Puikkonen, A., Kärkkäinen, T., Häkkilä, J.: Cool interaction with calm technologies - experimenting with ice as a multitouch surface. In: Proceedings of ITS 2010. ACM Press, pp. 15-18 (2010)

6. Geurts, L., Abeele, V.V.: Splash controllers: game controllers involving the uncareful manipulation of water. In: Proceedings of TEI 2012. ACM Press, pp. 183-186 (2012)

7. Pier, M.D., Goldberg, I.R.: Using water as interface media in VR applications. In Proceedings of CLIHC 2005. ACM Press, pp. 162-169 (2005)

8. Häkkilä, J., Koskenranta, O., Posti, M., He, Y.: City landmark as an interactive installation experiences with stone, water and public space. In: Proceedings of TEI 2014. ACM (2014)

9. Sodhi, R., Poupyrev, I., Glisson, M., Israr, A.: AIREAL: interactive tactile experiences in free air. ACM Trans. Graph. (TOG) 32(4), 134 (2013)

10. Rydarowski, A., Samanci, O., Mazalek, A.: Murmur: kinetic relief sculpture, multi-sensory display, listening machine. In: Proceedings of TEI 2008. ACM, pp. 231-238 (2008)

11. Juhlin, O., Zhang, Y., Sundbom, C., Fernaeus, Y.: Fashionable shape switching: explorations in outfit-centric design. In: Proceedings of CHI 2013. ACM, pp. 1353-1362 (2013)

12. Jung, H., Stolterman, E.: Material probe: exploring materiality of digital artifacts. In: Proceedings of TEI 2011. ACM Press, pp. 153-156 (2011)

13. Microsoft Product Reaction Cards. http://www.microsoft.com/usability/UEPostings/ ProductReactionCards.doc. Accessed 1 May 2015 